\title{
A Dementia Classification Framework using Frequency and Time-frequency Features based on EEG signals
}

\author{
Pholpat Durongbhan, Yifan Zhao, Senior Member, IEEE, Liangyu Chen, Panagiotis Zis, Matteo De \\ Marco, Zoe C. Unwin, Annalena Venneri, Xiongxiong He, Sheng Li, Yitian Zhao, Daniel J. \\ Blackburn, and Ptolemaios G. Sarrigiannis
}

\begin{abstract}
Alzheimer's Disease (AD) accounts for 60-70\% of all dementia cases, and clinical diagnosis at its early stage is extremely difficult. As several new drugs aiming to modify disease progression or alleviate symptoms are being developed, to assess their efficacy, novel robust biomarkers of brain function are urgently required. This study aims to explore a routine to gain such biomarkers using the quantitative analysis of Electroencephalography (QEEG). This paper proposes a supervised classification framework which uses EEG signals to classify healthy controls (HC) and AD participants. The framework consists of data augmentation, feature extraction, $\mathrm{K}$ Nearest Neighbour (KNN) classification, quantitative evaluation and topographic visualisation. Considering the human brain either as a stationary or a dynamical system, both frequency-based and time-frequency-based features were tested in $\mathbf{4 0}$ participants. Results: a) The proposed method can achieve up to $99 \%$ classification accuracy on short (4s) eyes open EEG epochs, with the KNN algorithm that has best performance when compared to alternative machine learning approaches; b) The features extracted using the wavelet transform produced better classification performance in comparison to the features based on FFT; c) In the spatial domain, the temporal and parietal areas offer the best distinction between healthy controls and AD. The proposed framework can effectively classify $\mathrm{HC}$ and AD participants with high accuracy, meanwhile offering identification and localisation of significant QEEG features. These important findings and the proposed classification framework could be used for the development of a biomarker for the diagnosis and monitoring of disease progression in $\mathrm{AD}$.
\end{abstract}

Index Terms-Electroencephalogram, Alzheimer's Disease, Machine Learning, K-Nearest Neighbour, Signal Processing.

\section{INTRODUCTION}

A lzheimer's disease (AD) is the most common form of dementia characterised by a cognitive decline that for the very elderly has to be greater than that expected by the normal ageing process [1]. It is characterised by multiple cognitive deficits, including memory decline, thinking, behaviour and more importantly deficits in carrying out everyday tasks [2]. Early detection of $\mathrm{AD}$ is challenging because many of the symptoms overlap with those of normal ageing related decline.

Pholpat Durongbhan and Yifan Zhao, School of Aerospace, Transport and Manufacturing, Cranfield University, Bedfordshire MK43 0AL, UK.

Liangyu Chen, corresponding author, Department of Neurosurgery, Shengjing Hospital of China Medical University, Shenyang, China.

Yitian Zhao, Cixi Institute of Biomedical Engineering, Ningbo Institute of Industrial Technology, Chinese Academy of Sciences, Ningbo, China
Currently, there is no disease-modifying therapeutic intervention for dementia due to $\mathrm{AD}$, but early detection would allow for better care and treatment planning for challenging behavioural and psychological symptoms [3]. Additionally, as several new treatments are undergoing evaluation in clinical trials, sensitive, non-invasive and reproducible biomarkers are urgently required to identify and recruit patients in the prodromal phase of the disease, to be implemented as objective outcomes measures and to monitor disease progression. In clinical practice, $\mathrm{AD}$ diagnosis is reached through a series of tests such as family history check, cognitive tests, along with other brain imaging and monitoring methods [4]. Methods such as Magnetic Resonance Imaging (MRI), Computed Tomography (CT), Positron Emission Tomography (PET), and, to a very lesser extent, Electroencephalogram (EEG) have been used to assist practitioners in the diagnostic process [4].

EEG consists of electrical signals collected from electrodes placed on the patient's scalp [5]. They represent the electrical activity of the brain at the time of recording; frequency and amplitude content vary according to the subject's biological state (e.g. awake versus sleep), mental state, age, disease process etc. EEG has become an important non-invasive clinical tool that has helped increase our understanding of brain network complexity and for the identification of areas of dysfunction [5], [6].

EEG studies in patients with AD have consistently shown abnormalities. Slowing of the posterior dominant rhythms and an increase in the slow wave activity is the most common feature on visual analysis of the recordings, [7] while there is a reduction in alpha and beta frequencies, although this latter characteristic is much more difficult to detect on empirical inspection of an EEG. Over recent decades, EEG spectral analysis provided the means for more objective estimation of the frequencies involved and to determine, to an extent, their spatial distribution. With this frequency domain approach, the power for different frequency bands from any EEG channels can be quantified. This quantitative analysis has confirmed the

Xiongxiong He and Sheng Li, Information Engineering, Zhejiang University of Technology, Hangzhou 310023, China

Panagiotis Zis, Daniel J. Blackburn, Matteo De Marco, Zoe C. Unwin, Annalena Venneri and Ptolemaios G. Sarrigiannis, Department of Neurosciences, Sheffield Teaching Hospitals, NHS Foundation Trust, Royal Hallamshire Hospital, Sheffield S10 2JF, UK. 
aforementioned observations from visual empirical EEG interpretation [7]. More specifically, it is generally thought that the earliest changes in $\mathrm{AD}$ are an increase in theta and a decrease in beta activity [7]. The prevailing view is that EEG abnormalities are associated with cognitive deficits, and this electro-clinical correlation is important [7], [8]. However, despite all this previous spectral analysis work none of these techniques is widely adopted and none, to our knowledge, is considered as a validated biomarker for clinical use, mainly due to low sensitivity and specificity. On the other hand, quantitative electroencephalography (QEEG) is gaining recognition as a means of monitoring brain function [9]. It has the potential to analyse in time, frequency, and time-frequency domains the electrical traces created by the dynamic emergence of neuronal workspaces, though to underpin cognitive functions [6]. QEEG can monitor brain activity through translating electrophysiological measures, with variable parametric and non-parametric frequency and time domain methodologies, into meaningful continuous numerical variables that can be then used to identify brain network deficits and/or as a source of surrogate endpoints in research [10]. It offers an objective approach next to the very useful but non-specific behavioural and cognitive performance assessments, commonly used as clinical diagnostic tools and as outcome measures for pharmaceutical trials. As a plethora of variables can be produced with distinctive QEEG methods, interpretation and classification of the results can become particularly cumbersome. Without the need for any model or test assumptions [11] the discipline of machine learning (ML) has found breeding ground in this field and numerous publications use this approach to demonstrate its potential to be implemented as an accurate method of identifying patients with $\mathrm{AD}$, for the differential diagnosis from other forms of dementia and as a source of surrogate outcome measures in trials involving subjects in the prodromal phase of disease [10], [12]-[17].

There are therefore, two dimensions to consider, on one end the QEEG analysis and the methods to extract features defined from EEG signals and the second, the selection of a ML technique appropriate to achieve an ideal cohort classification (e.g. dementia versus normal cognitive function) based on the aforementioned EEG metrics. There is yet to be an agreed consensus in the field as to which combination of signal processing methods, features selection, and ML techniques would consistently yield the best classification accuracy. Some popular linear features used are derivative parameters of: signal discrete wavelet transform [18], signal coherence [19], [20], and signal synchrony [21]. Derivatives of other non-linear features such as: spectral entropy, spectral roll-off, zerocrossing rate, [22], [23] correlation dimension, and Lyapunov exponent [24] had also been used, while some studies had proposed novel feature extraction method themselves such as Integrated Pattern Monitoring [25] and multi-channel deep convolutional neural network which combine feature extraction with classification processes [26]. In terms of ML techniques, a multitude of well-established methods have been employed with supervised learning algorithms being the most widespread. Support Vector Machine (SVM) was the most widely used technique [18], [22], [25], [27]. However, simpler supervised learning algorithms such as Linear and Quadratic Discriminant Analysis (LDA, QDA) [28], [29], logistic regression [23], classification tree and random forest [18], [28] had also been used. In some studies, clustering algorithms such as Gaussian Mixture Model (GMM) [20] was used to perform unsupervised classification.

A. In this work, we use a cohort of patients with $\mathrm{AD}$ and a group of healthy controls (HC) to assess the accuracy of various QEEG features and ML methods in distinguishing between these two diagnostic states and then develop a framework based on the highest performance technique. Although most studies in this field use eyes closed EEG epochs this paper shows that eyes open resting state EEG data (typically characterised by a desynchronised EEG in healthy asymptomatic controls) achieve significant classification accuracy. This paper additionally studies the impact of two different frequency domain analysis methods on the classification accuracy of a ML algorithm, a fast Fourier Transform (FFT) versus Wavelet Transforms, the latter also offer information on the dynamic behaviour of various frequency bands over time. It should be noted that the FFT approach considers the behaviour of the epoch as stationary while wavelet transform provides timesolved frequency response which is more appropriate for a dynamical system (details in C. Mother Wavelet Selection, supplementary material).

$B$. With regards to the software side of this work, one notable similarity among all previous research studies is that the data processing methodology can be separated into 4 distinct steps: signal pre-processing, application of feature extraction algorithm, application of ML algorithms, and result evaluation. For each new study, a new framework will have to be set up to handle the entire process. Presently, to the best of the authors' knowledge, there are robust EEG processing frameworks such as EEGLAB, but none is geared towards using machine learning in the study of neurodegenerative diseases. A properly organised framework with its implementation, specifically designed to handle EEG applications in a ML context, would greatly facilitate the pace at which new research could be produced and translated into a clinically useful informative biomarker about state and severity that could also be used in clinical trials [10]. Thus, a novel dementia classification framework was developed in this study and applied to EEG recordings after comparing various ML algorithms (details in B. Classification Approaches, supplementary material).

\section{Methodology}

\section{A. Case selection}

Participants were $\mathrm{HC}$ or patients diagnosed with AD who had detailed neuropsychology testing and structural and functional (fMRI) scans. All subjects were recruited from Sheffield Teaching Hospitals NHS Trust memory clinic, and HCs were enrolled through opportunity sampling and word of mouth over a period of a year (February 2015-16). Twenty HCs (10 younger than $70 \mathrm{y}$ old, 11 females, 10 older than $70 \mathrm{y}$ old with a mean age of $67 \mathrm{y}+/-\mathrm{SD}$ of $12 \mathrm{y}$ ) and $20 \mathrm{AD}$ cases (10 female, 16 younger than $70 \mathrm{y}$ old and 4 older than $70 \mathrm{y}$ old, mean age $64 \mathrm{y}+/$-SD of 
$8 y)$ were collected. Further details to include education, neuropsychology examinations and structural MRI findings are described in great detail in previously published work [30]. The diagnosis of $\mathrm{AD}$ was made according to NINCDS-ADRDA criteria [31] and based on a consensus taking into account clinical history, neurological examination, neuropsychological scores and neuroradiological findings. All participants provided informed consent and this project was approved by the Yorkshire and the Humber (Leeds West) Research Ethics Committee (reference number 14/YH/1070).

\section{B. EEG recordings}

\section{1) EEG acquisition}

We chose task free EEG that requires minimal cooperation as cognitive deficits in patients with $\mathrm{AD}$ render cognitive paradigms difficult to implement. This article is based on secondary analyses carried out on the same dataset used in previous work [18].

A modified 10/10 overlapping a 10/20 international system of electrode placement was adopted. An XLTEK 128-channel headbox (Optima Medical LTD) was used sampling at $2 \mathrm{~K} \mathrm{~Hz}$ (analogue low pass filter at $600 \mathrm{~Hz}$ ) with an earlobe reference (jump cables were devised to combine the right and left earlobe electrodes while impedances where kept equal between sides). Resting state, 30 minutes, EEG recordings were acquired; including distinct eyes open (EO) and eyes closed (EC) 5minute epochs (all participants were encouraged to rest and not think about anything specific). If they showed signs of drowsiness, they were prompted.

The data were categorised according to their age group, either below 70 or above 70 . For each subject, the data were collected twice in 2 physiological states: EO and EC. To investigate further the effect of age on the results, another 2 datasets were created by combining both age groups together but with different eye states. In these combined sets, equal number of subjects were selected from both age groups to prevent the combined group's results from being skewed towards the below 70 categories due to the larger number of samples of the below 70 . For each eye state, subjects from both age groups were selected randomly: $8 \mathrm{AD}$ (4 from each group) and $20 \mathrm{HC}$ (10 from each group). The 6 groups and their composition are summarised in Table I.

$$
\text { TABLE I }
$$

THE NUMBER OF SUBJECTS OF SIX STUDIED GROUPS

\begin{tabular}{lcc}
\hline \hline & AD & HC \\
\hline Below 70 EO & 16 & 10 \\
Above 70 EO & 4 & 10 \\
Combined EO & 8 & 20 \\
Below 70 EC & 16 & 10 \\
Above 70 EC & 4 & 10 \\
Combined EC & 8 & 20 \\
\hline \hline
\end{tabular}

\section{2) EEG data selection and artefacts removal}

All EEGs were reviewed with time-locked video recordings on an XLTEK equipment (Optima Medical LTD) with bipolar derivations. The following bipolar channels were available: Fp2-F8, Fp1-F7, F8-F4, F7-F3, F4-C4, F3-C3, F4-FZ, FZ-CZ,
F3-FZ, T4-C4, T3-C3, C4-CZ, C3-CZ, CZ-PZ, C4-P4, C3-P3, T4-T6, T3-T5, P4-PZ, P3-PZ, T6-O2, T5-O1, P4-O2, P3-O1, $\mathrm{O} 2-\mathrm{O} 1$. Both EO and EC artefact free epochs, each of 12 seconds in duration, were selected for analysis. To avoid bias, the first 12-s EO/EC epochs were used from each of the 40 participants. Spike 2 (version 8) software was used for data preprocessing and export, where a time constant $\tau=0.08 \mathrm{~s}$ (high pass filter at $2 \mathrm{~Hz}$ ) was applied to the data attenuating any delta or slower frequency artefacts, like those generated by eye blinking and movements.

\section{Classification Framework}

The proposed framework includes four steps: data augmentation, feature extraction, classification, and evaluation and visualisation. The framework was implemented in MATLAB2017 environment. The 4 steps were implemented in this framework as separate classes in the software.

\section{1) Data Augmentation}

In this study, the number of samples in all dataset is considered relatively small for ML applications. This issue is common among many studies which have less than 50 subjects in the dataset used [23]-[25], [27], [29], [32]. It is understood that data scarcity is a major issue in this field. Segmenting the data samples into smaller, but still informative, segments is a possible remedy for this issue and has been adopted in previous work [27], [32]. A data augmentation process is therefore proposed in this framework. It segments the signals into smaller data of equal length and gives the new data the same label as the original data. In this study, the EEG data is 12 seconds long. If the segment number is 2 , each segment would be 6 seconds long, and the total number of samples would be increased from 40 to 80 , with the same $\mathrm{AD}$ to $\mathrm{HC}$ ratio. The software implementation has a built-in data segmentation functionality. The user would simply have to state the signal length and the desired number of segments.

\section{2) Feature Extraction}

Any numerical parameters can be defined as features in this framework, and they are key to determine classification performance. In this paper, features are defined according to 5 frequency bands of interest: delta band $(0-4 \mathrm{~Hz})$, theta band (4$8 \mathrm{~Hz})$, alpha band $(8-12 \mathrm{~Hz})$, beta band $(12-30 \mathrm{~Hz})$, and gamma band $(30-50 \mathrm{~Hz})$. Since lower delta band $(0-2 \mathrm{~Hz})$ components had been filtered out in the previous process, delta band frequencies used in this study would be confined to the $2-4 \mathrm{~Hz}$ range only. Furthermore, it should be noted that before the features are extracted, the framework would pass all data through a 10th-order low pass Butterworth filter with a cut-off frequency at $50 \mathrm{~Hz}$, to remove higher frequency components. This lowpass filtering was done because artefacts from muscle activity, which mainly consist of high frequencies up to $300 \mathrm{~Hz}$, may contaminate the EEG signals. Without this step, it would be difficult to determine if the effect observed in the highfrequency spectrum is of neural or muscle origin [33].

The feature extraction functionality is implemented as an abstract class interface where new features could be defined in a new derived class with its own properties. This allows new features to be implemented while still maintaining 
compatibility with the remaining framework. Two types of features were of interest in this study which are based on the frequency domain and time-frequency domain respectively.

The FFT features $\left(F F_{i}\right)$ used in this study were defined as the average magnitude of all FFT coefficients in a specific frequency band, which can be written as:

$$
F F_{i}=\frac{\sum_{k=f s_{i}}^{f e_{i}}\left|X_{k}\right|}{f e_{i}-f s_{i}},
$$

where $f s_{i}$ is the starting frequency of the $i^{t h}$ band, $f e_{i}$ is the ending frequency, and $X_{k}$ are the FFT coefficients. If the human brain behaviour is simplified as a stationary system, this feature represents the averaged frequency response at each band.

The Continuous Wavelet Transform (CWT) features $\left(W F_{i}\right)$ used in this study were defined as the average magnitude of all CWT coefficients, obtained using a selected mother wavelet, in that particular frequency band over the entire signal length, which can be written as:

$$
W F_{i}=\frac{\sum_{j=t s}^{t e} \Sigma_{k=f s_{i}}^{f e_{i}}\left|Y_{j, k}\right|}{(t e-t s)\left(f e_{i}-f s_{i}\right)}
$$

where $f s_{i}$ is the starting frequency of the $i^{\text {th }}$ band, $f e_{i}$ is the ending frequency, $t s$ is the starting time, te is the ending time, and $Y_{j, k}$ are the wavelet transform coefficients based on the selected mother wavelet. If the human brain is considered as a non-stationary or dynamic system, where the behaviour is time dependent, these features can better represent the frequency response at each band. This study employed various mother wavelet and concluded that the bump mother wavelet produces the best performance (details in C. Mother Wavelet Selection, supplementary material).

Both FFT and CWT features were extracted and assessed in terms of the performance on classifying $\mathrm{AD}$ patients from $\mathrm{HC}$ participants and gain new insights into how the location of channels and frequency bands correlate to $\mathrm{AD}$ classification accuracy. Such studies are important to understand better the spatial and frequency distribution of brain network deficits in AD. The studies conducted to assess their performance are as follows:

Individual Channel analysis: The objective of this study is to inspect how well the selected features extracted from each individual EEG channel can be used to train an $\mathrm{AD}$ vs $\mathrm{HC}$ classifier. In this study, each of the 23 channels is used to train the classification model separately. Since each channel represents a spatial area on the scalp, the result of this study would suggest how indicative each channel is when it comes to $\mathrm{AD}$ diagnosis.

Individual Frequency Band analysis: The objective of this study is to inspect how well a single frequency band can be used to classify $\mathrm{AD}$ and $\mathrm{HC}$ subjects. Analogous to the previous study, each channel is used to train the model individually; however, instead of using all frequency bands, only 1 frequency band was used as the feature. The results would reveal which frequency band at which channel would be indicative of $\mathrm{AD}$.

3) Classification

The framework implemented the K-Nearest Neighbour (KNN) classification method with 10 -fold cross-validation as the default method in the Classification class. The class is implemented with an interface enforcing compatibility with the Feature class. Other ML classification algorithms can also be used by changing the classification function within the class. The training process is conducted repeatedly on the same data with a certain number of iterations (50 as the default value). The performance of the 50 models trained in this process is then evaluated and averaged. This is done due to the random nature of how ML models are trained with the cross-validation process. Averaging results over several iterations would lower the fluctuation and provide a better measure of how well the method performed, in general.

\section{4) Evaluation and Visualisation}

Cross-validation is employed in this paper to evaluate the classification performance. The data were initially divided into $N$ folders, and $N-1$ folders are then selected for the training and the remaining folder is for the testing. This step is repeated for $N$ times until each folder has been used for the testing, and the accuracy is finally averaged. Cross-validation combines measures of fitness in prediction to derive a more accurate estimate of model prediction performance [34]. In this paper, 10 -fold cross-validation was used.

To evaluate the performance of the classification model trained in the previous process, the following parameters are calculated and evaluated:

- $\quad \operatorname{Loss}(\mathrm{L})$

$$
L=\frac{F P+F N}{T P+T N+F P+F N}
$$

- Accuracy (Acc)

$$
A c c=1-\text { Loss }
$$

- $\quad$ Sensitivity (Sens)

$$
\text { Sens }=\frac{T P}{T P+F N}
$$

- $\quad$ Specificity (Spec)

$$
\text { Spec }=\frac{T N}{T N+F P}
$$

where True Positive (TP): number of AD sample classified correctly by the algorithm as AD; False Positive (FP): number of $\mathrm{HC}$ sample classified incorrectly by the algorithm as $\mathrm{AD}$; True Negative (TN): number of HC sample classified correctly by the algorithm as $\mathrm{HC}$; False Negative (FN): number of $\mathrm{AD}$ sample classified incorrectly by the algorithm as HC.

In this framework, a visualisation method was developed based on an eegplot function developed by Ikaro Silva [35]. The visualisation method is used to create a topographic scalp map by interpolating values from each electrode position. In this framework, the values plotted are normalised first. This is because of the different nature of the frequency magnitude in different frequency bands, and normalising these values would provide a more user-friendly plot in terms of relative magnitudes. This paper develops a customised implementation of eegplot by incorporating this study's electrode positions into the method so that the coordinates will not have to be manually selected every time when the method is called. For the provided dataset, the channel locations have been pre-coded and are represented by the black dots in the topographic plot result, as shown in Fig. 1. Each channel's location and name can be seen when overlaid with the electrode diagram. 


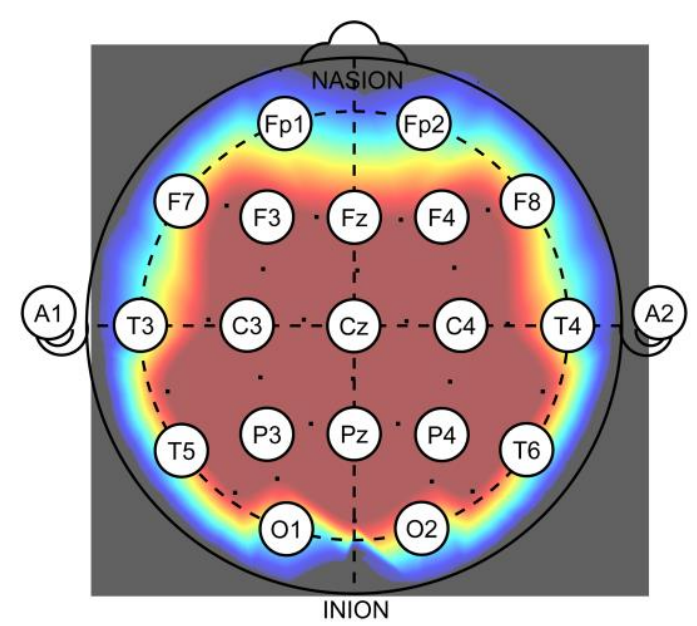

Fig. 1. Topographic plot result overlaid with EEG channel location diagram. Black dots correspond to the electrode locations for dataset used in this study.

\section{RESULTS}

To select the optimal parameters of each step, a sensitivity analysis was conducted and the details are shown in the Supplementary Material. The results below were produced using the following parameters: three segments per sample in data augmentation, $\mathrm{KNN}(\mathrm{K}=2)$ for classification, bump mother wavelet for CWT.

\section{A. Overall Classification Accuracy}

The average classification results for both features can be seen in Table II. It is noticeable that, for all groups of datasets, the classification algorithm achieved high average classification accuracy ( $>78 \%$ for FFT and $>80 \%$ for CWT). For the below 70 groups, in particular, it shows reasonably high sensitivity (>89\% for FFT and $>90 \%$ for CWT) and specificity ( $>81 \%$ for FFT and $>83 \%$ for CWT) values compared to other datasets. However, for the above 70 groups, the sensitivity values dropped significantly ( $>64 \%$ for FFT and $>67 \%$ for CWT) while the specificity values remained comparable (>84\% for FFT and $>85 \%$ for CWT) to those of the below 70 groups.

This trend can still be seen when both age groups were combined while keeping their EO/EC states separated. The average accuracy and sensitivity values dropped when

$$
\text { TABLE II }
$$

THE AVERAGE CLASSIFICATION ACCURACY, SENSITIVITY, AND SPECIFICITY

\begin{tabular}{ccccccc}
\multicolumn{2}{c}{ AMONG ALL CHANNELS IN EACH DATA SET. (IN \%, MEAN(SD)) } \\
\hline \hline \multirow{2}{*}{ Dataset } & \multicolumn{2}{c}{ Accuracy } & \multicolumn{2}{c}{ Sensitivity } & \multicolumn{2}{c}{ Specificity } \\
& FFT & CWT & FFT & CWT & FFT & CWT \\
\hline \multirow{2}{*}{ Below 70 EO } & 85.94 & 87.61 & 89.08 & 90.90 & 81.48 & 83.04 \\
& $(4.34)$ & $(4.84)$ & $(3.82)$ & $(4.52)$ & $(6.30)$ & $(6.49)$ \\
Above 70 EO & 83.09 & 85.68 & 72.33 & 77.68 & 86.89 & 89.00 \\
& $(6.85)$ & $(5.98)$ & $(12.59)$ & $(12.29)$ & $(5.70)$ & $(4.68)$ \\
Combined EO & 82.30 & 83.32 & 70.75 & 72.57 & 86.59 & 87.52 \\
& $(4.11)$ & $(3.96)$ & $(6.99)$ & $(7.85)$ & $(4.03)$ & $(3.16)$ \\
Below 70 EC & 88.73 & 90.26 & 91.91 & 93.00 & 84.35 & 86.43 \\
& $(6.46)$ & $(5.45)$ & $(4.69)$ & $(4.40)$ & $(8.89)$ & $(7.57)$ \\
Above 70 EC & 78.66 & 80.30 & 64.86 & 67.73 & 84.01 & 85.10 \\
& $(5.60)$ & $(4.52)$ & $(11.71)$ & $(9.26)$ & $(3.71)$ & $(3.60)$ \\
Combined EC & 82.23 & 83.41 & 73.30 & 73.80 & 86.78 & 86.89 \\
& $(4.50)$ & $(4.40)$ & $(8.71)$ & $(8.70)$ & $(3.32)$ & $(3.42)$ \\
\hline \hline
\end{tabular}

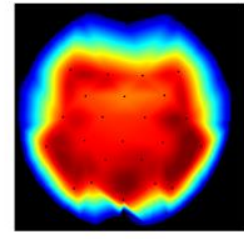

a) Below $70 \mathrm{EO}$

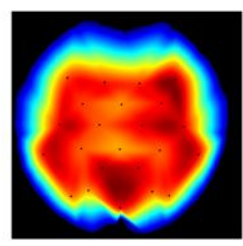

c) Above $70 \mathrm{EO}$

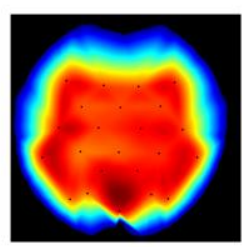

e) Combined EO

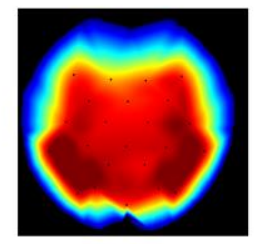

b) Below $70 \mathrm{EC}$

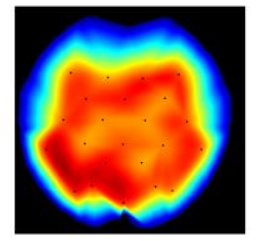

d) Above $70 \mathrm{EC}$

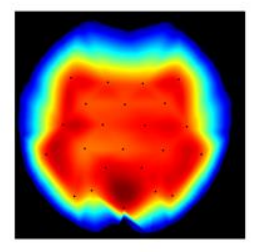

f) Combined EC

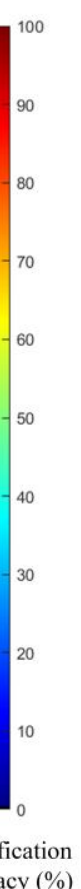

Accuracy $(\%)$

Fig. 2. Scalp topographic plot of normalised classification accuracy obtained from using individual channel using FFT features.

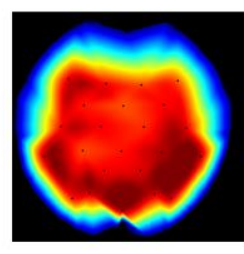

a) Below $70 \mathrm{EO}$

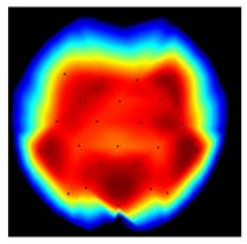

c) Above $70 \mathrm{EO}$

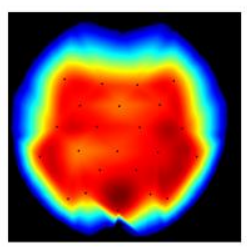

e) Combined EO

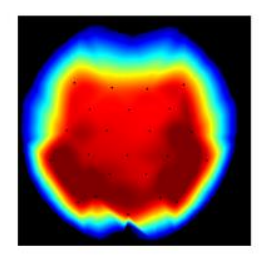

b) Below $70 \mathrm{EC}$

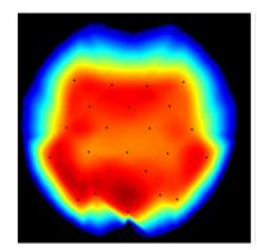

d) Above $70 \mathrm{EC}$

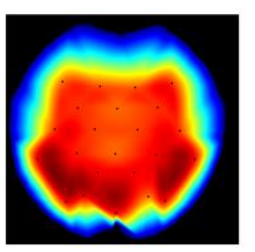

f) Combined EC

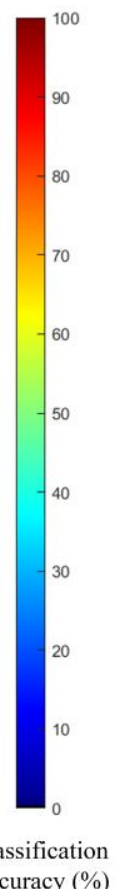

Accuracy $(\%)$
Fig. 3. Scalp topographic plot of normalised classification accuracy obtained from using individual channel using CWT features.

compared to the below 70 datasets while remained higher than the above 70 sets alone. It is suspected that the data from the above 70 groups are causing this drop while data from the below 70 groups are cushioning the magnitude of this drop in the combined sets. 

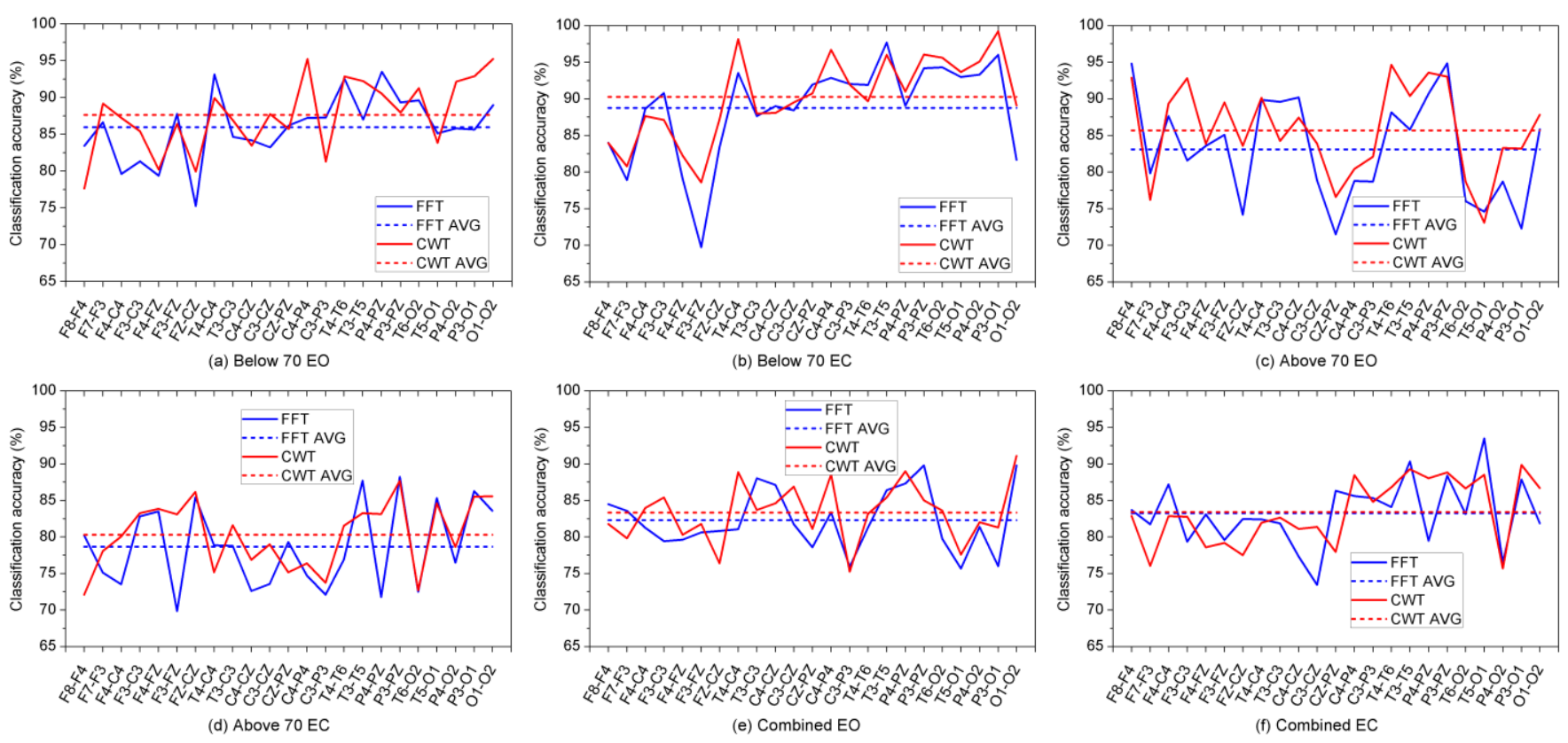

Fig. 4. CWT and FFT accuracy comparison for all channels in different datasets.

\section{B. Classification Accuracy for Each Channel}

The resulting classification accuracy for each channel and dataset were plotted in a topographic map in the evaluation process. These results can be seen in Fig. 2 for FFT features and Fig. 3 for CWT features, where the classification accuracy (in percentage) for each dataset was plotted. The colour bar on the right provides a reference guide to the values in the plots. Darker red zones illustrated area whose features produced high classification accuracy, while lighter areas' features produced relatively lower accuracy. The classification accuracy values for each channel as well as its average for each feature type (FFT or CWT) for all datasets are also plotted in Fig. 4 to provide further overview on the topographically plotted results.

From Fig. 2 and 3, it can be noticed in all groups that the temporal (T-channels), parietal (P-channels), and occipital (Ochannels) areas produced relatively high classification accuracy.

It can also be seen across all age groups that the classification accuracy changes highly in the frontal area (F-channels) when comparing between EO and EC states. This change is the most prominent in the frontal area of the above 70 groups where the frontal area with high classification accuracy can be observed in the EO state, but not in the EC state.

\section{Channels with High Classification Accuracy}

The performance of each channel is evaluated, and the top five channels with the highest accuracy for each group are shown in Table III. For all below 70 groups, the top 5 channels have achieved very high classification accuracy at around $90 \%$ and higher for both FFT and CWT features. This is in line with the overall average result for both groups presented earlier. Even though the overall accuracy of the above 70 groups is low, when each channel's performance was considered separately, very high accuracy ( $>85 \%$ for FFT and $>84 \%$ for CWT) was achieved. The fact that these channels in the above $70 \mathrm{EO}$
TABLE III

THE TOP 5 CHANNELS AND ITS CLASSIFICATION ACCURACY FOR EACH DATASET. (IN \%, *ACC DENOTES ACCURACY)

\begin{tabular}{|c|c|c|c|c|c|c|c|}
\hline \multicolumn{4}{|c|}{ Below 70 EO } & \multicolumn{4}{|c|}{ Below 70 EC } \\
\hline \multicolumn{2}{|c|}{ FFT } & \multicolumn{2}{|c|}{ CWT } & \multicolumn{2}{|c|}{ FFT } & \multicolumn{2}{|c|}{ CWT } \\
\hline Channel & Acc & Channel & Acc & Channel & Acc & Channel & Acc \\
\hline 'P4-PZ' & 93.49 & 'C4-P4' & 95.26 & 'T3-T5' & 97.69 & 'P3-O1' & 99.23 \\
\hline 'T4-C4' & 93.15 & '01-o2' & 95.26 & 'P3-O1' & 96.00 & 'T4-C4' & 98.15 \\
\hline 'T4-T6' & 92.54 & 'P3-O1' & 92.90 & 'T6-O2' & 94.28 & 'C4-P4' & 96.67 \\
\hline 'T6-O2' & 89.59 & 'T4-T6' & 92.87 & 'P3-PZ' & 94.15 & 'P3-PZ' & 96.02 \\
\hline 'P3-PZ' & 89.33 & 'T3-T5' & 92.21 & 'T4-C4' & 93.51 & 'T3-T5' & 96.00 \\
\hline \multicolumn{4}{|c|}{ Above 70 EO } & \multicolumn{4}{|c|}{ Above 70 EC } \\
\hline \multicolumn{2}{|c|}{ FFT } & \multicolumn{2}{|c|}{ CWT } & \multicolumn{2}{|c|}{ FFT } & \multicolumn{2}{|c|}{ CWT } \\
\hline Channel & Acc & Channel & Acc & Channel & Acc & Channel & Acc \\
\hline 'P3-PZ' & 94.86 & 'T4-T6' & 94.67 & 'P3-PZ' & 88.24 & 'P3-PZ' & 88.67 \\
\hline 'F8-F4' & 94.81 & 'P4-PZ' & 93.57 & 'T3-T5' & 87.71 & 'FZ-CZ' & 86.14 \\
\hline 'P4-PZ' & 90.71 & 'P3-PZ' & 93.00 & 'P3-O1' & 86.29 & 'O1-O2' & 85.57 \\
\hline 'C4-CZ' & 90.19 & 'F8-F4' & 92.90 & 'FZ-CZ' & 85.43 & 'P3-O1' & 85.52 \\
\hline 'T4-C4' & 89.86 & 'F3-C3' & 92.81 & 'T5-O1' & 85.33 & 'T5-O1' & 84.62 \\
\hline \multicolumn{4}{|c|}{ Combined EO } & \multicolumn{4}{|c|}{ Combined EC } \\
\hline \multicolumn{2}{|c|}{ FFT } & \multicolumn{2}{|c|}{ CWT } & \multicolumn{2}{|c|}{ FFT } & \multicolumn{2}{|c|}{ CWT } \\
\hline Channel & Acc & Channel & Acc & Channel & Acc & Channel & Acc \\
\hline 'P3-PZ' & 89.79 & 'O1-O2' & 91.10 & 'T5-O1' & 93.48 & 'P3-O1' & 89.86 \\
\hline 'O1-O2' & 89.76 & 'P4-PZ' & 89.00 & 'T3-T5' & 90.33 & 'T3-T5' & 89.29 \\
\hline 'T3-C3' & 88.02 & 'T4-C4' & 88.86 & 'P3-PZ' & 88.38 & 'P3-PZ' & 88.83 \\
\hline 'P4-PZ' & 87.33 & 'C4-P4' & 88.55 & 'P3-O1' & 87.88 & 'T5-O1' & 88.50 \\
\hline 'C4-CZ' & 87.10 & 'C3-CZ' & 86.88 & 'F4-C4' & 87.19 & 'C4-P4' & 88.45 \\
\hline
\end{tabular}

groups have high accuracy in contrast to its overall values would suggest that the overall drop in accuracy was heavily contributed by other channels. While the accuracy was high (>89\% for FFT and $>92 \%$ for CWT) in the above 70 EO dataset, the accuracy dropped significantly (dropped to $>85 \%$ for FFT and $84 \%$ for CWT) when the eye state was changed to EC. 
TABLE IV

THE TOP 5 CHANNELS BASED ON THE AVERAGE CLASSIFICATION ACCURACY OVER ALL DATASET. (IN \%, MEAN(SD))

FFT

CWT

\begin{tabular}{cccc} 
Channel Name & Average Accuracy & Channel Name & Average Accuracy \\
\hline 'P3-PZ' & $90.79(2.87)$ & 'P3-PZ' & $89.75(3.67)$ \\
'T3-T5' & $89.15(4.08)$ & 'T3-T5' & $89.42(4.21)$ \\
'T4-C4' & $86.47(5.91)$ & 'O1-O2' & $89.25(3.21)$ \\
'T4-T6' & $85.81(5.63)$ & 'P4-PZ' & $89.21(3.22)$ \\
'P4-PZ' & $85.30(7.44)$ & 'P3-O1' & $88.66(6.13)$ \\
\hline \hline
\end{tabular}

TABLE V

THE AVERAGE CLASSIFICATION ACCURACY FOR EACH FREQUENCY BAND AND DATASET. (IN \%, MEAN(SD))

\begin{tabular}{|c|c|c|c|c|c|c|}
\hline & & $\delta$ & $\theta$ & $\alpha$ & $\beta$ & $\gamma$ \\
\hline \multirow{7}{*}{ 岳 } & Below 70 EO & $\begin{array}{l}57.62 \\
(8.40)\end{array}$ & $\begin{array}{l}62.87 \\
(4.81)\end{array}$ & $\begin{array}{l}58.04 \\
(5.43)\end{array}$ & $\begin{array}{l}63.16 \\
(7.85)\end{array}$ & $\begin{array}{c}60.42 \\
(7.46)\end{array}$ \\
\hline & Below 70 EC & $\begin{array}{l}60.29 \\
(5.10)\end{array}$ & $\begin{array}{l}66.85 \\
(4.43)\end{array}$ & $\begin{array}{l}54.47 \\
(6.63)\end{array}$ & $\begin{array}{l}61.85 \\
(7.93)\end{array}$ & $\begin{array}{l}56.10 \\
(8.83)\end{array}$ \\
\hline & Above $70 \mathrm{EO}$ & $\begin{array}{l}66.64 \\
(6.50)\end{array}$ & $\begin{array}{l}75.28 \\
(9.04)\end{array}$ & $\begin{array}{l}66.69 \\
(7.63)\end{array}$ & $\begin{array}{l}68.48 \\
(7.99)\end{array}$ & $\begin{array}{l}72.40 \\
(7.91)\end{array}$ \\
\hline & Above 70 EC & $\begin{array}{c}66.36 \\
(10.10)\end{array}$ & $\begin{array}{l}71.04 \\
(7.92)\end{array}$ & $\begin{array}{l}63.26 \\
(8.35)\end{array}$ & $\begin{array}{l}65.54 \\
(6.58)\end{array}$ & $\begin{array}{l}66.15 \\
(7.06)\end{array}$ \\
\hline & Combined EO & $\begin{array}{l}67.12 \\
(4.77)\end{array}$ & $\begin{array}{l}69.12 \\
(6.87)\end{array}$ & $\begin{array}{l}62.07 \\
(4.89)\end{array}$ & $\begin{array}{l}64.22 \\
(5.70)\end{array}$ & $\begin{array}{l}65.50 \\
(6.26)\end{array}$ \\
\hline & Combined EC & $\begin{array}{l}68.02 \\
(6.98) \\
\end{array}$ & $\begin{array}{l}69.10 \\
(6.99) \\
\end{array}$ & $\begin{array}{l}59.42 \\
(4.47) \\
\end{array}$ & $\begin{array}{l}63.35 \\
(5.41)\end{array}$ & $\begin{array}{r}61.86 \\
(5.13) \\
\end{array}$ \\
\hline & Average & $\begin{array}{l}64.34 \\
(3.92)\end{array}$ & $\begin{array}{l}69.04 \\
(3.78)\end{array}$ & $\begin{array}{l}64.43 \\
(3.91)\end{array}$ & $\begin{array}{l}60.66 \\
(2.13)\end{array}$ & $\begin{array}{l}63.74 \\
(5.11)\end{array}$ \\
\hline \multirow{7}{*}{ 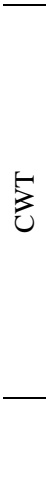 } & Below 70 EO & $\begin{array}{l}57.69 \\
(6.11)\end{array}$ & $\begin{array}{c}60.03 \\
(8.75)\end{array}$ & $\begin{array}{l}57.80 \\
(7.34)\end{array}$ & $\begin{array}{l}55.12 \\
(6.21)\end{array}$ & $\begin{array}{l}56.63 \\
(7.27)\end{array}$ \\
\hline & Below 70 EC & $\begin{array}{l}58.64 \\
(6.05)\end{array}$ & $\begin{array}{l}59.42 \\
(7.36)\end{array}$ & $\begin{array}{l}52.12 \\
(7.20)\end{array}$ & $\begin{array}{l}54.40 \\
(6.82)\end{array}$ & $\begin{array}{l}52.17 \\
(6.31)\end{array}$ \\
\hline & Above 70 EO & $\begin{array}{c}64.67 \\
(10.43)\end{array}$ & $\begin{array}{l}67.39 \\
(8.90)\end{array}$ & $\begin{array}{c}63.25 \\
(10.96)\end{array}$ & $\begin{array}{l}60.77 \\
(7.36)\end{array}$ & $\begin{array}{l}58.28 \\
(8.06)\end{array}$ \\
\hline & Above $70 \mathrm{EC}$ & $\begin{array}{c}61.90 \\
(10.37)\end{array}$ & $\begin{array}{l}69.15 \\
(8.20)\end{array}$ & $\begin{array}{l}64.18 \\
(7.31)\end{array}$ & $\begin{array}{l}59.11 \\
(7.52)\end{array}$ & $\begin{array}{c}56.34 \\
(9.00)\end{array}$ \\
\hline & Combined EO & $\begin{array}{l}61.08 \\
(5.33)\end{array}$ & $\begin{array}{l}64.75 \\
(6.26)\end{array}$ & $\begin{array}{l}62.06 \\
(5.75)\end{array}$ & $\begin{array}{l}59.89 \\
(6.44)\end{array}$ & $\begin{array}{l}60.56 \\
(5.42)\end{array}$ \\
\hline & Combined EC & $\begin{array}{l}63.82 \\
(5.12)\end{array}$ & $\begin{array}{l}63.30 \\
(6.74)\end{array}$ & $\begin{array}{l}59.00 \\
(5.96)\end{array}$ & $\begin{array}{l}58.49 \\
(5.81)\end{array}$ & $\begin{array}{r}58.23 \\
(6.62) \\
\end{array}$ \\
\hline & Average & $\begin{array}{l}61.30 \\
(2.53)\end{array}$ & $\begin{array}{l}64.01 \\
(3.55)\end{array}$ & $\begin{array}{l}59.74 \\
(4.08)\end{array}$ & $\begin{array}{l}57.96 \\
(2.38)\end{array}$ & $\begin{array}{l}57.03 \\
(2.57)\end{array}$ \\
\hline
\end{tabular}

When each channel's accuracy was averaged over all datasets, the top 5 channels with the highest average accuracy is summarised in Table IV. From previous observations in Table II, it was noted that, overall, CWT features produce a higher average classification accuracy. Upon closer inspection of Table IV, however, the average accuracy of the top channels for CWT features are slightly lower than FFT features.

Furthermore, it can be observed from Table III and IV that, in general, the highest performing channels are mostly concentrated in the temporal (T-channels) and parietal (Pchannels) areas of the scalp, and to a lesser degree, the central (C-channels) and occipital (O-channels) areas in line with earlier observations based on Fig. 2 and 3. This is an encouraging result because these areas are the least affected by ocular artefacts commonly contaminating with artefact anterior, frontal EEG recordings (F-channels). Thus, it can be inferred that the resulting high accuracy was minimally affected by ocular artefacts. Moreover, these areas are also well known to be affected in $\mathrm{AD}$, a statement which these observations would further support [36].

\section{Classification Accuracy for Each Frequency Band}

The average classification accuracy for all channels when the average magnitudes of each frequency band were used to train the classification model are listed in Table V. Overall when only a single frequency band was used as features, the resulting accuracy was very low compared to using 5 bands together as features. For both FFT and CWT features, average accuracy values for single frequency features would hover around 50\% to $70 \%$ as opposed to $70 \%$ to $90 \%$ for all 5 features.

Closer inspection of Table V would reveal that, for both FFT and CWT features, delta and theta bands have consistently produced relatively high average accuracy with FFT features producing slightly higher average accuracy than CWT features. It is also worth noting that, relative to other datasets in the same study, the average accuracy obtained for the above 70 age group for every frequency band in this study is higher, this contrasts with the data shown in Table II where the below 70 group demonstrates higher average accuracy.

In Fig. 5 and 6, the average magnitude of the 5 frequency bands and their resulting classification accuracy were selected for further visualisation and analysis. The magnitude of FFT and CWT coefficients in each specific band was averaged and plotted separately for $\mathrm{HC}$ and AD participants in Fig. 5 and 6 for FFT and CWT features respectively. Another topographic plot of the accuracy obtained from models trained using that corresponding band is also placed below to facilitate the interpretability of the results.

In both FFT and CWT cases, the magnitudes of the coefficients and its location on the scalp change significantly from $\mathrm{HC}$ to $\mathrm{AD}$ participants. The most significant changes are observed in the EC state for both age groups and show reduced magnitudes in the frontal area for delta, theta, and alpha bands for HC. Some changes in average magnitudes can be observed in the EO state, but this is not as pronounced as the EEG is typically desynchronised in this biological state.

In a similar manner to Fig. 2 and 3, Fig. 5 and 6 also reveal that signals from channels in temporal, parietal, and occipital areas have high classification accuracy. However, the large changes in magnitude observed in Fig. 5 and 6 do not seem to translate into a higher classification accuracy for these channels. This can be appreciated in the magnitude plots where significant differences do not always correlate directly with the classification accuracy plots below them. It is possible that the fluctuations in magnitude which yield high accuracies for the temporal, parietal, and occipital areas are subtler but more consistent and patterned than the large changes observed in the frontal areas which might have been more irregular in nature.

\section{DISCUSSION}

The novelty of this work lies in the development of a new and robust software framework which has proven to be effective in improving classification of EEG recording and flexible in selecting the feature extraction methods and ML approaches. Its robustness is demonstrated in how 2 feature 


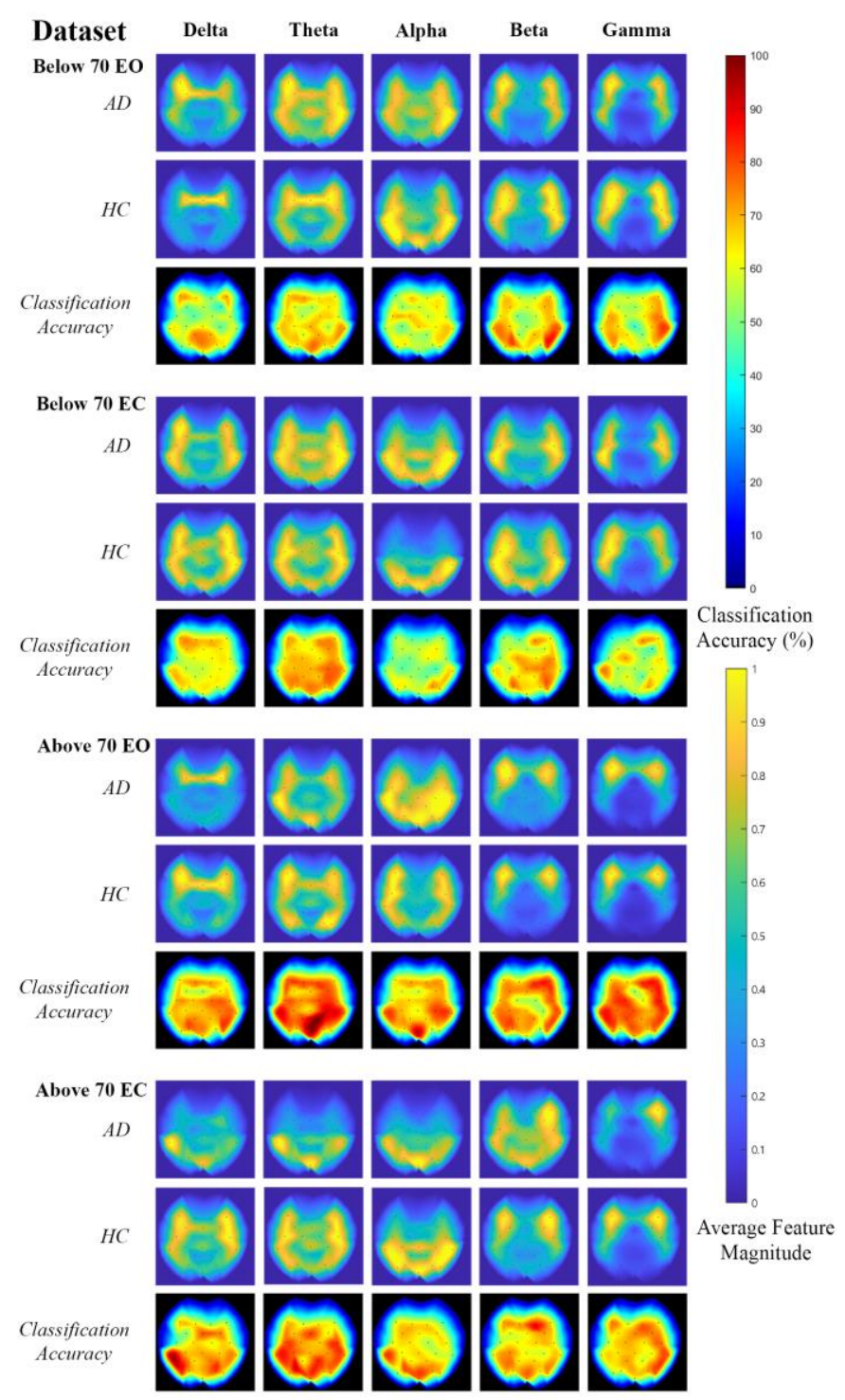

Fig. 5. Topographic plots displaying the average magnitude of FFT coefficients of selected frequency and their corresponding classification accuracy.

extractions, Fourier and Wavelet, were conducted using the framework. It has the potential to allow researchers to explore, not only various EEG features but also their ability to act as classifying parameters with minimal modification to the existing framework. As a matter of fact, with some modifications, its utility could be expanded to cover various brain disorders, other than dementia, and the potential to perform multi-class dataset classification could be implemented to separate between normal brain function and focal or generalised brain deficits underpinning various neurological disorders.

With regards to the studies conducted using this novel framework, we clearly distinguish between EO and EC EEG epochs and demonstrate different classification accuracies, significantly favouring the former state for the above 70 cohorts (95\% for EO Vs $85 \%$ EC) and slightly the latter for participants below 70 years of age (99\% for EC Vs $95 \%$ EO), shown in Table III. This raises the suspicion that due to age-related modifications in brain networks, the EO vs EC state appears to

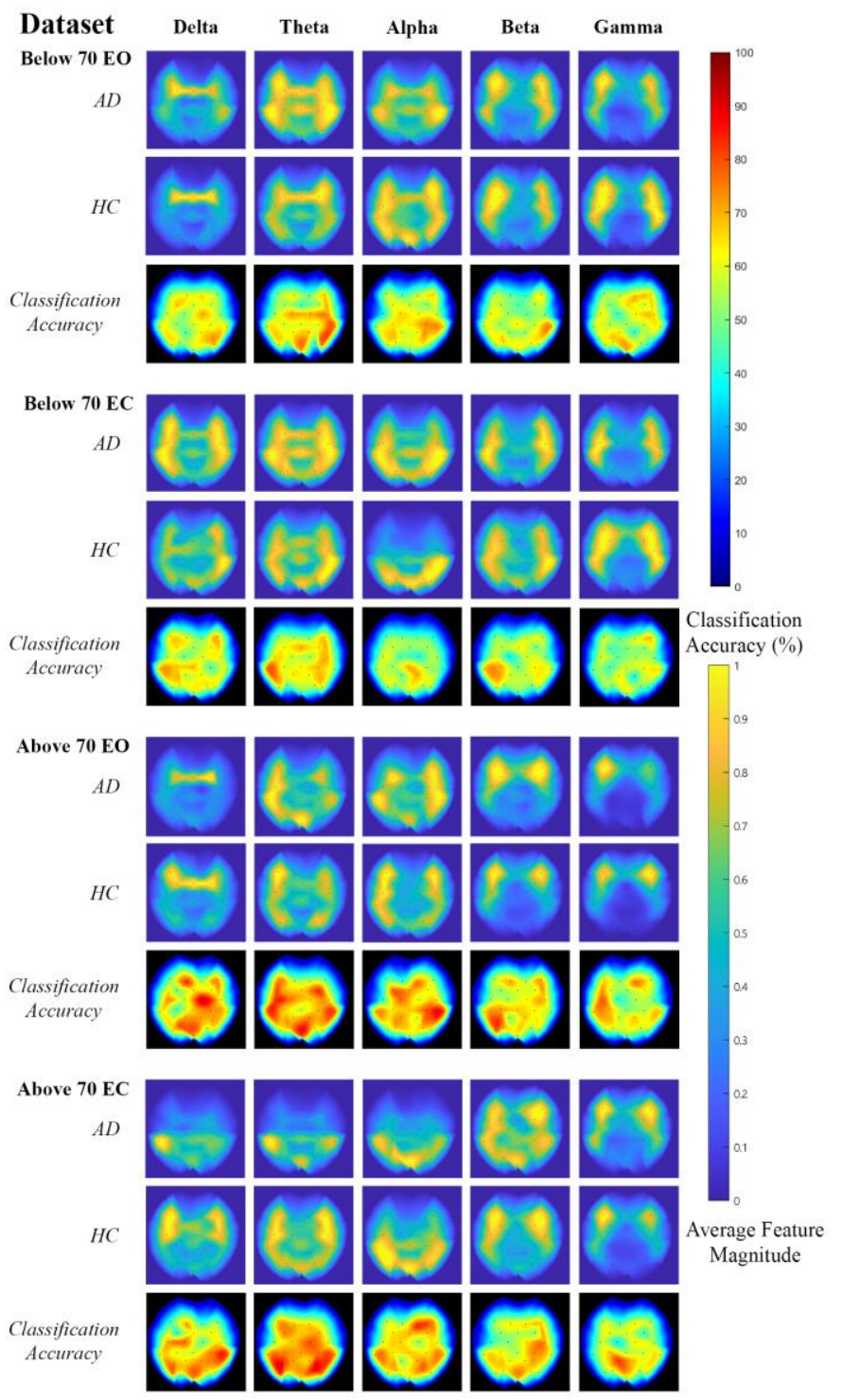

Fig. 6. Topographic plots displaying the average magnitude of CWT coefficients of selected frequency and their corresponding classification accuracy.

be more discriminating for those above the age of 70 , but improves accuracy in all age groups.

The low average sensitivity and accuracy in the above 70 groups could lead to the inference that ageing causes a general increase in randomness in frequency components in people who are over 70y of age. This random change in frequency characteristics would cause the classifier to be unable to detect a regular cluster or pattern from using frequency features which would result in its failure to produce high sensitivity models for participants above the age of 70 .

Equally of note in this study is the effect imposed by EEG data segmentation on classification accuracy. This shows that 4 second EEG mini-epochs offer the best trade-off between the number of samples on one hand (the 12-second epoch of each participant produces 3 mini-epochs) and the preservation of information that can be extrapolated from each EEG data segment on the other. When the length of the mini-epochs drops below 4 seconds, this translates into a gradual drop in performance (Fig. S1). Remarkably, EEG epochs of 4s each 
(8000 data points at a sampling rate of $2 \mathrm{KHz}$ ) produce a very high degree of classification accuracy above $90 \%$ (Fig. S1).

The performance of different ML algorithms, including Fine Classification Tree, Fine KNN, and the Fine Linear SVM were independently tested for the FFT and CWT features. Consistently the KNN method showed the best overall classification accuracy (Fig. S2). This difference in performance between methods is even more apparent when the CWT features were used (Fig. S2).

Despite their popularity in many studies, frequency and timefrequency characteristics still need further investigation. This validation study used simple feature definition in small numbers and has yielded up to $99 \%$ accuracy. This has demonstrated that complex feature definitions in large numbers are not necessary to produce high classification accuracy.

One main drawback of the validation studies is the fact that the features were defined based on average values of the Fourier and wavelet transform coefficients. These features provide a good overview of the frequency domain but do not give any temporal information. This means that the research particularly with the FFT approach largely disregards the time component of the features and treats the brain as a linear and static system. $\mathrm{KNN}$ itself is a non-parametric and non-linear classification method, and the study did not take advantage of its full potential. To capitalise on these characteristics fully, other features containing temporal information, such as those offered by wavelet-based cross-spectrum and bispectrum estimates and other parametric time domain methods like the Error reduction ratio causality test [37] should be considered as well in future work. Clearly larger cohorts of age-stratified patients with different forms of dementia and healthy controls need to be studied within the KNN algorithm, an ideally suited nonlinear ML method to be applied in this type of multi-class classification problem.

\section{CONCLUSION}

In this research, a novel dementia classification framework based on EEG data has been developed and implemented in MATLAB 2017 environment, to classify patients with Alzheimer's Disease from healthy control participants using signal processing and ML algorithms.

The results have demonstrated that the framework's process can reach a classification accuracy of up to $97 \%$ for Fourier transform features and $99 \%$ for wavelet transform features for some channels (Table III). The framework's numerical and visual result has also shown that channels around the temporal, parietal, and occipital areas have consistently high classification accuracy for Alzheimer's Disease in keeping with previous studies [7]. This spatial distribution of the abnormalities is consistent in both eye states, hinting that this is independent of the subject's eye state (EO/EC). This finding is also reproduced in the age combined datasets, suggesting that the topology of the findings is additionally age independent and consistent with previous research criteria that identify the temporoparietal areas as typically involved in AD pathology [36].

The results also have indicated that delta and theta band features produced the highest average classification accuracy. This is in keeping with previous observations that have identified slowing of the EEG in Alzheimer's Disease, with an increase of theta and delta power and good correlation between the degree of these EEG abnormalities and cognitive impairment [7]. Therefore, this shift of neuronal synchronisation over low frequency bands in this type of dementia appears to disrupt information processing in cortical networks, as it is associated to the cognitive dysfunction [7]. Further studies focusing on these two frequencies should be conducted in future work.

In conclusion, the framework developed and its subsequent validation results have shown that this approach is robust and flexible and can be used in studies involving EEG data, signal processing techniques, and ML classification algorithms in dementia research. The validation studies have also provided new insights into how channels and frequency bands can be used to pinpoint specific areas and parameters which could be used to develop AD biomarkers. The developed framework shows a high classification accuracy with EEG epochs as short as 4 seconds each, highlighting the amount of hidden information embedded within routinely performed EEG recordings, that can be easily and readily obtained at low cost. This study contributes to the definition of a methodology that is highly efficient at identifying the presence of $\mathrm{AD}$ when the disease is in its moderate stage. This method paves the way towards its application during earlier disease stages (i.e., at the mild cognitive impairment stage).

\section{ACKNOWLEDGEMENTS}

This work was supported in part by the Through-Life Engineering Services Centre at Cranfield University (UK), in part by Royal Hallamshire Hospital at Sheffield (UK), and in part by the Computational and Software Techniques in Engineering MSc Course at Cranfield University (UK). This research carried out in part at the National Institute for Health Research (NIHR) Sheffield Biomedical Research Centre (Translational Neuroscience)/NIHR Sheffield Clinical Research Facility. We would like to thank Neurocare (charitable fund) for purchasing the EEG equipment used for the recordings in this work. This work is also supported by the Liaoning Science and Technology Plan Project (20180550047), Shenyang Science and Technology Plan Project (18-013-0-58) and Shengjing Hospital Project (MF45). For access to the code and data underlying this paper, please see the Cranfield University repository, CORD, at: https://doi.org/ 10.17862/cranfield.rd.7673702

\section{REFERENCES}

[1] E. Kravitz, J. Schmeidler, and M. S. Beeri, "Cognitive decline and dementia in the oldest-old.," Rambam Maimonides Med. J., vol. 3, no. 4, p. e0026, Oct. 2012

[2] World Health Organization, "Fact Sheet: Dementia," 2017. [Online]. Available: http://www.who.int/en/news-room/factsheets/detail/dementia. [Accessed: 01-Oct-2018].

[3] World Health Organization, "Dementia: a public health priority," World Health Organization, 2012

[4] National Health Service, "Tests for diagnosing dementia - NHS," 
2017. [Online]. Available:

https://www.nhs.uk/conditions/dementia/diagnosis-tests/. [Accessed: 21-Sep-2018].

[5] L. Sörnmo and P. Laguna, Bioelectrical signal processing in cardiac and neurological applications. Elsevier Academic Press, 2005.

[6] F. Lopes da Silva, "EEG and MEG: relevance to neuroscience," Neuron, vol. 80, no. 5, pp. 1112-1128, 2013.

[7] J. Jeong, "EEG dynamics in patients with Alzheimer's disease," Clin. Neurophysiol., vol. 115, no. 7, pp. 1490-1505, Jul. 2004.

[8] R. P. Brenner et al., "Computerized EEG spectral analysis in elderly normal, demented and depressed subjects," Electroencephalogr Clin Neurophysiol, vol. 64, no. 6, pp. 483-492, 1986.

[9] E. C. van Straaten, P. Scheltens, A. A. Gouw, and C. J. Stam, "Eyesclosed task-free electroencephalography in clinical trials for Alzheimer's disease: an emerging method based upon brain dynamics," Alzheimers Res Ther, vol. 6, no. 9, p. 86, 2014.

[10] S. Simpraga et al., "EEG machine learning for accurate detection of cholinergic intervention and Alzheimer's disease," Sci Rep, vol. 7, no. 1, p. 5775, 2017.

[11] G. Shmueli, "To Explain or to Predict?," Stat. Sci., vol. 25, no. 3, pp. 289-310, Aug. 2010.

[12] C. Lehmann et al., "Application and comparison of classification algorithms for recognition of Alzheimer's disease in electrical brain activity (EEG)," J Neurosci Methods, vol. 161, no. 2, pp. 342-350, 2007.

[13] L. R. Trambaiolli, A. C. Lorena, F. J. Fraga, P. A. M. Kanda, R. Anghinah, and R. Nitrini, "Improving Alzheimer's Disease Diagnosis with Machine Learning Techniques," Clin. Eeg Neurosci., vol. 42, no. 3, pp. 160-165, 2011.

[14] Z. J. Dai et al., "Discriminative analysis of early Alzheimer's disease using multi-modal imaging and multi-level characterization with multi-classifier (M3)," Neuroimage, vol. 59, no. 3, pp. 2187-2195, 2012.

[15] V. Podgorelec, "Analyzing EEG Signals with Machine Learning for Diagnosing Alzheimer's Disease," Elektron. Ir Elektrotechnika, vol. 18, no. 8, pp. 61-64, 2012.

[16] A. So, D. Hooshyar, K. W. Park, and H. S. Lim, "Early Diagnosis of Dementia from Clinical Data by Machine Learning Techniques," Appl. Sci., vol. 7, no. 7, 2017.

[17] N. Houmani et al., "Diagnosis of Alzheimer's disease with Electroencephalography in a differential framework," PLoS One, vol. 13, no. 3, 2018.

[18] G. Fiscon et al., "Alzheimer's disease patients classification through EEG signals processing," in 2014 IEEE Symposium on Computational Intelligence and Data Mining (CIDM), 2014, pp. $105-112$.

[19] K. Akrofi, M. C. Baker, M. W. O’Boyle, and R. B. Schiffer, "Clustering and modeling of EEG coherence features of Alzheimer's and mild cognitive impairment patients," in 2008 30th Annual International Conference of the IEEE Engineering in Medicine and Biology Society, 2008, pp. 1092-1095.

[20] K. Akrofi, R. Pal, M. C. Baker, B. S. Nutter, and R. W. Schiffer, "Classification of Alzheimer's disease and mild cognitive impairment by pattern recognition of EEG power and coherence," in 2010 IEEE International Conference on Acoustics, Speech and Signal Processing, 2010, pp. 606-609.

[21] J. Dauwels, F. Vialatte, C. Latchoumane, Jaeseung Jeong, and A. Cichocki, "EEG synchrony analysis for early diagnosis of Alzheimer's disease: A study with several synchrony measures and EEG data sets," in 2009 Annual International Conference of the IEEE Engineering in Medicine and Biology Society, 2009, pp. 2224 2227.

[22] T. Staudinger and R. Polikar, "Analysis of complexity based EEG features for the diagnosis of Alzheimer's disease," in 2011 Annual International Conference of the IEEE Engineering in Medicine and Biology Society, 2011, vol. 2011, pp. 2033-2036.

[23] C. Gomez et al., "Bispectral analysis of spontaneous EEG activity from patients with moderate dementia due to Alzheimer's disease," in 2017 39th Annual International Conference of the IEEE Engineering in Medicine and Biology Society (EMBC), 2017, pp. 422-425.
[24] J. Jeong, J. H. Chae, S. Y. Kim, and S. H. Han, "Nonlinear dynamic analysis of the EEG in patients with Alzheimer's disease and vascular dementia.," J. Clin. Neurophysiol., vol. 18, no. 1, pp. 58-67, Jan. 2001.

[25] A. Nancy, M. Balamurugan, and S. Vijaykumar, "A brain EEG classification system for the mild cognitive impairment analysis," in 2017 4th International Conference on Advanced Computing and Communication Systems (ICACCS), 2017, pp. 1-6.

[26] F. C. Morabito et al., "Deep convolutional neural networks for classification of mild cognitive impaired and Alzheimer's disease patients from scalp EEG recordings," in 2016 IEEE 2nd International Forum on Research and Technologies for Society and Industry Leveraging a better tomorrow (RTSI), 2016, pp. 1-6.

[27] L. R. Trambaiolli, N. Spolaôr, A. C. Lorena, R. Anghinah, and J. R. Sato, "Feature selection before EEG classification supports the diagnosis of Alzheimer's disease," Clin. Neurophysiol., vol. 128, no. 10, pp. 2058-2067, Oct. 2017.

[28] M. Dauwan et al., "Random forest to differentiate dementia with Lewy bodies from Alzheimer's disease.," Alzheimer's Dement. (Amsterdam, Netherlands), vol. 4, pp. 99-106, 2016.

[29] P. M. Rodrigues, B. C. Bispo, D. R. Freitas, J. P. Teixeira, and A. Carreres, "Evaluation of EEG spectral features in alzheimer disease discrimination," in European Signal Processing Conference, 2013, pp. $1-5$.

[30] D. Blackburn et al., "A Pilot Study Investigating a Novel Non-Linear Measure of Eyes Open versus Eyes Closed EEG Synchronization in People with Alzheimer's Disease and Healthy Controls," Brain Sci., vol. 8, no. 7, p. 134, Jul. 2018.

[31] B. Dubois et al., "Research criteria for the diagnosis of Alzheimer's disease: revising the NINCDS-ADRDA criteria," Lancet Neurol, vol. 6, no. 8, pp. 734-746, 2007.

[32] W. S. Pritchard et al., "EEG-based, neural-net predictive classification of Alzheimer's disease versus control subjects is augmented by non-linear EEG measures.," Electroencephalogr. Clin. Neurophysiol., vol. 91, no. 2, pp. 118-30, Aug. 1994.

[33] S. D. Muthukumaraswamy, "High-frequency brain activity and muscle artifacts in MEG/EEG: a review and recommendations," Front. Hum. Neurosci., vol. 7, p. 138, Apr. 2013.

[34] G. Seni and J. F. Elder, "Ensemble Methods in Data Mining: Improving Accuracy Through Combining Predictions," Synth. Lect. Data Min. Knowl. Discov., vol. 2, no. 1, pp. 1-126, Jan. 2010.

[35] Ikaro Silva, "eegplot - File Exchange - MATLAB Central," 2013. [Online]. Available: https://uk.mathworks.com/matlabcentral/fileexchange/3279-eegplot. [Accessed: 21-Sep-2018].

[36] B. Dubois et al., "Advancing research diagnostic criteria for Alzheimer's disease: the IWG-2 criteria," Lancet Neurol, vol. 13, no. 6, pp. 614-629, 2014.

[37] Y. Zhao, S. A. Billings, H. Wei, and P. G. Sarrigiannis, “Tracking time-varying causality and directionality of information flow using an error reduction ratio test with applications to electroencephalography data," Phys Rev E Stat Nonlin Soft Matter Phys, vol. 86, no. 5 Pt 1, p. 51919, 2012. 


\title{
A dementia classification framework using frequency and time-frequency features based on EEG signals
}

\author{
Durongbhan, Pholpat
}

IEEE

Durongbhan P, Zhao Y, Chen L, Zis P, De Marco M, Unwin JC, Venneri A, He X, Li Sheng, Zhao Y, Blackburn DJ and Sarrigiannis PG., A dementia classification framework using frequency and time-frequency features based on EEG signals, IEEE Transactions on Neural Systems and Rehabilitation Engineering, Volume 27, Issue number 5, pp. 826-835.

https://doi.org/10.1109/TNSRE.2019.2909100

Downloaded from Cranfield Library Services E-Repository 